\title{
¿Existe un régimen internacional para la defensa y promoción de la democracia?
}

\section{Resumen}

La defensa y promoción de la democracia constituye una preocupación central de la política exterior de muchos de los actores del sistema internacional, como Estados Unidos, la Unión Europea y diversos países y agrupaciones regionales en el resto del mundo. En el continente americano, la Organización de Estados Americanos (OEA) adoptó en el año 2001 la Carta Democrática Interamericana, instrumento que constituye el núcleo central del "Régimen Democrático Interamericano" y que pretende promover y defender la plena vigencia de la democracia en las Américas. ¿¿Hasta qué punto estos intereses e iniciativas configuran un nuevo régimen internacional?

El artículo concluye que la promoción de la democracia forma parte de una tradición política liberal y occidental y que por consiguiente su universalización y transformación en un régimen global dependerá de la, por ahora poco probable, expansión de esa misma tradición al resto del mundo. Mientras ello no suceda y mientras subsistan distintas concepciones de lo que constituye un orden justo y legítimo en materia política, no sólo a nivel global sino que también a nivel regional, resultará difícil hablar de un régimen internacional preciso y efectivo para la defensa de la democracia.

\footnotetext{
*Profesor Titular, Facultad de Derecho, Universidad de Chile. Las opiniones expresadas en este artículo se emiten a título personal y sólo comprometen al autor.
}

\begin{abstract}
The defense and promotion of democracy represents a basic concern of the foreign policy of many actors in the international system, including the United States, the European Union and several countries and regional groupings in the rest of the world. In the Americas, the Organization of American state (OAS) adopted in 2001 the Interamerican Democratic Charter, an instrument which constitutes the core of the "Interamerican Democratic Regime" and which pretends to promote and defend the full enforcement of democracy in the region. To what extent these interests and initiatives embody a new international regime?

The article concludes that the promotion of democracy is part of a Western liberal political tradition and, consequently, its universalization and transformation in a global
\end{abstract}


regime will depend on the, by now unlikely, expansion of this tradition in the rest of the world. As long as there are different notions about what represents a legitimate and just political order, not only at the global but also at the regional level, it will be difficult to speak of a precise and effective international regime for the defense of democracy.

\section{El concepto de régimen internacional}

Antiguamente, el derecho internacional reservaba el concepto de régimen internacional para un determinado estatuto territorial, aplicable por ejemplo a ríos, canales o territorios dotados de estatutos especiales como las islas Äland, Danzig o Trieste. Asimismo, un régimen normalmente tenía su origen en tratados o convenciones ${ }^{1}$. En la actualidad, el concepto se ha ampliado y tiene un carácter más funcional, referido por ejemplo a derechos humanos, el medio ambiente o el desarme, y puede originarse tanto en tratados como en reglas de otra naturaleza e incluso conductas. El Profesor Schachter los identifica como mecanismos colaborativos por medio de los cuales los Estados regulan un área particular de actividad, destacando su carácter institucional y su función regulatoria ${ }^{2}$.

Esta conceptualización se acerca a la noción de régimen internacional que surgió en la década de 1980 entre especialistas norteamericanos de las relaciones internacionales, permitiendo un diálogo más activo entre politólogos y juristas. Definido por los primeros como un conjunto, explícito o implícito, de principios, normas, reglas y procedimientos de toma de decisiones, alrededor de los cuales convergen las expectativas de los actores en un área determinada de la política internacional ${ }^{3}$, su remisión, al menos parcial, al ámbito jurídico se hace evidente. Aunque el concepto de régimen internacional tiene una acepción más restringida en el derecho internacional ${ }^{4}$, se puede definir como un conjunto de normas, primarias y secundarias, que regulan una cierta área de la actividad internacional, que no están necesariamente sometidas a la regla de la reciprocidad y que incluso pueden vincular a los Estados que no han consentido en su aprobación 5 .

Los regímenes internacionales se basan en reglas e instituciones, pero pueden ir más allá como consecuencia de las exigencias de coordinación, incluyendo por ejemplo

\footnotetext{
Shinja Murase, International Law. An integrative Perspective of Transboundary Issues, Tokyo: Sophia University Press, 2011, pp. $132-133$.

2 Oscar Schachter, International Law in Theory and Practice, Dordrecht y Boston: Nijhoff Publishers, 1991, pp. $74-76$.

3 Stephen D. Krasner, "Structural Causes and Regime Consequences: Regimes as Intervening Variables”, en: Beth A. Simmons y Richard H. Steinberg (eds.), International Law and International Relations, Cambridge: Cambridge University Press, 2006, p. 3.

4 Para un buen resumen del debate jurídico en este ámbito, véase el artículo de Oriol Casanovas y La Rosa, "Aproximación a una teoría de los regímenes en derecho internacional público", en Ángel J. Rodrigo y Caterina García (eds.), Unidad y Pluralismo en el Derecho Internacional Público y en la Comunidad Internacional, Coloquio en homenaje a Oriol Casanovas, Madrid: Tecnos, 2011, pp. 41-60.

Basado libremente en Casanovas, ibid., pp. 47 -51.
} 
prácticas y decisiones informales, orientaciones, directrices y prácticas de facto que se alejan de normas y que buscan la realización de objetivos funcionales. En la medida en que avanza la globalización, los regímenes regulatorios se van haciendo menos nacionales y más internacionales, menos domésticos y más universales. El fenómeno es claro en materias comerciales, pero también se hace evidente en muchas otras áreas, como los derechos humanos, el medio ambiente, el desarme, los derechos de los pueblos indígenas, etc. Naturalmente, la globalización se extiende al ámbito jurídico y, cuando se habla de regularla o de domesticarla, se está pensando en la adopción de principios, normas, reglas y procedimientos en las múltiples áreas en que ello se considera necesario. De esta manera, los regímenes se van desnacionalizando e internacionalizando de manera gradual y variable. Por otra parte, como lo demuestra la experiencia reciente y no tan reciente en temas tan diversos como el derecho internacional humanitario, la regulación medioambiental, los derechos humanos, el derecho comercial o el desarme, la participación en la elaboración de regímenes no se restringe sólo a Estados sino que incluye también a organizaciones internacionales, organizaciones no gubernamentales (ONG) y también a actores privados.

El desarrollo de nuevos regímenes y la transformación de los antiguos no han estado exentos de problemas. Muchas veces, los límites de estos regímenes son difusos. En ocasiones, se hace evidente una colisión de regímenes, que responden a lógicas políticas diferentes. Para citar un ejemplo frecuente y que ya ha dado lugar a una jurisprudencia frondosa aunque no siempre unívoca, se puede mencionar la colisión entre los regímenes de libre comercio y aquellos relativos a la protección del medio ambiente. A veces, las disputas entre regímenes diversos son dirimidas por la misma instancia, pero en otros casos pueden existir instancias alternativas o paralelas. La estructura descentralizada del orden internacional y del sistema jurídico que lo apoya, facilita este fenómeno. La expansión del derecho internacional y la multiplicación de instancias jurisdiccionales de carácter funcional o regional, han agudizado el problema de la fragmentación del derecho internacional, tema que ha sido objeto de tratamiento por la Comisión de Derecho Internacional de las Naciones Unidas ${ }^{6}$. En su informe, la Comisión alude al surgimiento de interpretaciones diversas del derecho internacional general, al surgimiento de excepciones institucionalizadas al derecho internacional general, al conflicto entre distintos regímenes legales y a las diferencias en los procesos de adopción de normas internacionales. Aunque existen criterios para la solución de estos problemas, como la especificidad (lex specialis), la temporalidad (lex posterior), la jerarquía normativa (ius cogens, obligaciones erga omnes), el criterio de la interpretación sistémica (artículo 31, N³ letra c) de la Convención de Viena sobre Derecho de los Tratados) o la prevalencia de las obligaciones impuestas por la Carta de las Naciones Unidas (artículo 103 de la Carta), su aplicación tampoco está

\footnotetext{
Informe y conclusiones del Grupo de Estudio sobre Fragmentación del Derecho Internacional: dificultades derivadas de la diversificación y expansión del Derecho internacional, $58^{\circ}$ período de sesiones celebrado del 1 de mayo al 9 de junio y del 3 de julio al 11 de agosto de 2006. Informe de la Comisión de Derecho Internacional, Suplemento $N^{\circ} 10$ (A/61/10), Capítulo XII, párrafos 241 -251, pp. 441 -465.
} 
exenta de dificultades y contradicciones ${ }^{7}$. Con todo, hay un cierto consenso en estimar que pese a esta fragmentación, que refleja en parte la diversidad y pluralismo de la comunidad internacional, se mantiene la unidad del derecho internacional, a través de la universalización del contenido de sus normas fundamentales, de la búsqueda de una cierta uniformidad en las soluciones sustantivas y a la adaptación de las normas y contenidos a los desarrollos recientes y las necesidades de la comunidad internacional ${ }^{8}$.

Los regímenes internacionales admiten múltiples clasificaciones. Pueden ser formales o informales, altamente institucionalizados o poco institucionalizados, pueden coincidir con organizaciones internacionales o incluir a varias de éstas, pueden ser regionales o universales, pueden agruparse en categorías funcionales (derechos humanos, medio ambiente, comercio, inversión, finanzas, recursos naturales, desarme, seguridad colectiva, asuntos marítimos, lucha contra la corrupción, etc.), pueden ser rígidos o laxos, estables o evolutivos. No es éste el lugar para profundizar o agotar estas clasificaciones. Más bien, nos interesa identificar un área específica, la protección y promoción de la democracia, para evaluar si estamos o no frente a un régimen internacional.

\section{La defensa de la democracia como régimen}

La defensa y promoción de la democracia constituye una preocupación central de la política exterior de muchos de los actores del sistema internacional. Tres presidentes estadounidenses de muy distinta orientación -Clinton, Bush y Obama- la han identificado como uno de los objetivos principales de la política exterior de los Estados Unidos. Los países de la Unión Europea (UE), actuando tanto a título individual como en el contexto de las instituciones comunitarias, han subrayado su compromiso con la defensa y difusión de la democracia en el mundo.

El consenso es menor en Asia y África. En muchos países del antiguo Tercer Mundo se tiende a relativizar la vigencia de ciertos derechos humanos o se invocan condiciones políticas y culturales especiales que justificarían enfoques singulares en esta materia. El viejo argumento sobre la primacía de los derechos económicos y sociales o del derecho al desarrollo por sobre las libertades políticas o personales, también conserva su popularidad en esas áreas. Aun así, varios países asiáticos y africanos se han sumado a los esfuerzos que se despliegan en favor de un nuevo régimen internacional para la protección de los derechos humanos y la defensa de la democracia. La Unión Africana incluyó la democracia entre sus principios básicos y tiene atribuciones para condenar golpes de Estado en los países miembros e incluso puede expulsar a gobiernos surgidos de manera no democrática. Sin embargo, en la práctica, ha utilizado estas atribuciones de manera selectiva.

Casanovas, "Aproximación a una teoría", pp. $42-43$.

Ángel Rodrigo Hernández, "La integración normativa y la unidad del derecho internacional público", en Rodrigo y García, Unidad y Pluralismo en el Derecho Internacional, p. 323. 
La existencia de situaciones que pueden conducir a Estados fallidos ha provocado igualmente un mayor activismo internacional a favor de los procesos de democratización. Así, la restauración del gobierno democrático de Haití constituyó la justificación oficial para la constitución de una (primera) fuerza de paz de Naciones Unidas en Haití, como lo declaró expresamente la Resolución 940 (1994) del Consejo de Seguridad que la autorizó. La intervención de las Naciones Unidas para restaurar la democracia en ese país, adoptada bajo el Capítulo VII de la Carta de la ONU, representó la primera vez que una crisis interna en que, en esencia, estaba en juego la recuperación democrática, provocaba medidas tan drásticas por parte del órgano máximo de la organización mundial?

En el continente americano, la democracia representa una de las piedras angulares del sistema interamericano y actuó como uno de los principios básicos para el establecimiento del Grupo de Río, el mecanismo de concertación política regional que se estableció en América Latina en 1986 y que dio lugar el 2010 a la Comunidad de Estados Latinoamericanos y Caribeños (CELAC). Si en el pasado la reivindicación democrática tenía un carácter más bien retórico y mediante un uso manifiestamente parcial fue convertida en un arma de la guerra fría, en las últimas décadas ha dado lugar a acciones colectivas. El "Compromiso de Santiago con la Democracia y la Renovación del Sistema Interamericano” y la Resolución 1080 sobre "Democracia Representativa”, adoptados por la XXI Asamblea General de la Organización de Estados Americanos (OEA), representaron hitos importantes en esta dirección, desde el momento en que estos instrumentos establecieron un mecanismo de respuesta frente a situaciones de interrupción ilegal del proceso democrático en cualquier nación de la OEA. En la práctica, el Grupo de Río siguió durante años una línea similar, pese a la resistencia de varios de sus miembros, llegando incluso a suspender a aquellos países miembros en que se registró una interrupción del proceso democrático.

La OEA adoptó la Carta Democrática Interamericana en el año 2001, instrumento que constituye el "núcleo central del Régimen Democrático Interamericano"10, que pretende promover y defender la plena vigencia de la democracia en las Américas.

En esta ponencia cabe preguntarse hasta qué punto esta voluntad política se ha traducido en un nuevo régimen jurídico internacional para la protección y promoción de la democracia. Una revisión somera de los principales manuales de derecho internacional no arroja muchas luces en la materia; a lo más, se encuentran referencias al tema en los capítulos sobre la protección internacional de los derechos humanos, el principio de libre determinación o autodeterminación de los pueblos o el reconocimiento de los Estados, pero no hay un tratamiento sistemático y singular del tema y la cobertura que recibe es excepcional ${ }^{11}$. La literatura sobre regímenes internacionales tampoco es muy

\footnotetext{
Roland Rich, "Bringing Democracy into International Law", Journal of Democracy 12:3, julio 2001, pp. 20 -34. Beatriz M. Ramacciotti, Democracia y Derecho Internacional en las Américas. Córdoba, Argentina: Lerner, 2010, p. 175.

1 Entre las excepciones, cabe destacar a Antonio Cassese, International Law, $2^{\text {nd }}$ ed., Oxford: Oxford University Press, 2005, p. 395; Hugo Llanos Mansilla, Teoría y Práctica del Derecho Internacional Público. Tomo III. La persona humana ante el derecho internacional. Santiago: Ed. Jurídica, 2008, pp. 203 -208 y Edmundo Vargas Carreño, Derecho Internacional Público. De acuerdo a las normas y prácticas que rigen en el siglo XXI, Santiago: Ed. Jurídica de Chile, 2007, pp. $268-272$.
} 
expresiva en esta materia. En una compilación muy extensa de artículos publicados en la prestigiosa revista International Organization sobre regímenes internacionales, no se incluyó un capítulo sobre el tema ${ }^{12}$. Los volúmenes que se refieren a la política del derecho internacional y el surgimiento de nuevos órdenes normativos tampoco profundizan el asunto. Y aunque hay buenos estudios sobre la defensa internacional de la democracia ${ }^{13}$, el tema todavía no se incorpora al corpus central del derecho internacional.

En realidad, estamos frente a un régimen embrionario. Si bien éste ya tiene una cierta expresión jurídica, no es comparable con los regímenes que se han concretado en áreas como el comercio, el medio ambiente, los derechos humanos, el desarme, etc. Este régimen consistiría en un derecho, reconocido internacionalmente, a la democracia electoral, que se basa en los derechos humanos, pero que se extiende para asegurar la participación significativa de los gobernados en las decisiones políticas formales que los afectan ${ }^{14}$. Los orígenes del régimen se encontrarían en el principio de libre determinación de los pueblos, aplicado en el proceso de descolonización consagrado en la Carta de las Naciones Unidas (Capítulos XI y XII), en el Pacto Internacional de Derechos Civiles y Políticos que entró en vigor en 1976 (especialmente su artículo 25), en diversas resoluciones de la Asamblea General y de la antigua Comisión y actual Consejo de Derechos Humanos de las Naciones Unidas, en las Cartas fundacionales, resoluciones y prácticas de diversas organizaciones regionales, en protocolos de convenciones regionales y en sentencias de tribunales regionales de derechos humanos.

\section{Los instrumentos}

La práctica en este ámbito también apunta a un régimen internacional en formación. Los actores interesados en la promoción y defensa de la democracia a nivel internacional están recurriendo a una cantidad creciente de medios o instrumentos para alcanzar este objetivo. Muchos de estos medios tienen un efecto incierto y, como recuerda Whitehead $^{15}$, pueden ser mutuamente inconsistentes y resultan de difícil aplicación durante un período muy prolongado.

En una primera categoría de instrumentos se puede situar la acción declarativa en todas sus variantes. Ella incluye las declaraciones de autoridades gubernamentales, especialmente cuando se producen golpes de Estado o amenazas hacia el orden constitucional;

12 Simmons y Steinberg, International Law, op. cit.

13 Véase por ejemplo Thomas M. Franck, "The Emerging Right to Democratic Governance", American Journal of International Law 86 (1992), pp. 49 -52; Tom Farer (ed), Beyond Sovereignty. Collectively Defending Democracy in the Americas. Baltimore: The Johns Hopkins University Press, 1996, y Gregory H. Fox y Brad D. Roth (eds.), Democratic Governance and International Law, Cambridge: Cambridge University Press, 2000; Ramac. Desde una perspectiva más politológica, véase Heraldo Muńoz (ed.), Democracy Rising. Assessing the Global Challenges. Boulder: Lynne Rienner, 2006.

14 Thomas M. Franck, "Legitimacy and the democratic entitlement", en Fox y Roth, Democratic Governance, p. 26.

15 Whitehead, Laurence, "International Aspects of Democratization”. En: Guillermo O’Donnell, Philippe C. Schmitter y Laurence Whitehead (Comps.), Transitions form Authoritarian Rule. Comparative Perspectives. Baltimore: The Johns Hopkins University Press, 1986, p. 19. 
la publicación de informes sobre incumplimiento de prácticas democráticas en otros países, normalmente asociadas con violaciones de los derechos humanos; la expresión, oral o escrita, pública o privada, de interés en determinados casos nacionales; las recomendaciones de comisiones nacionales y otras acciones similares. Estas expresiones de preocupación e interés pueden provenir de fuentes gubernamentales de muy variable nivel, de organizaciones regionales o globales, de grupos de Estados y de un amplio espectro de organizaciones no gubernamentales $(\mathrm{ONG})$ e incluso personalidades privadas.

La práctica internacional incluye múltiples ejemplos del uso de estos instrumentos y demuestra la variedad de resultados que se pueden alcanzar. Sin perjuicio de esta variación, queda claro que si bien estos medios no suelen producir efectos dramáticos y difícilmente podrían bastar por sí mismos para provocar un cambio de un régimen autoritario o permitir el asentamiento de uno democrático, representan pasos significativos para el logro de estos objetivos. Esta generalización es sobre todo válida para áreas regionales como América o Europa, pero se ha extendido también a África y a Asia. Si esta conclusión necesariamente general no fuera válida, no tendrían razón de ser las enérgicas reacciones que estas expresiones de preocupación provocan en los regímenes afectados. En un mundo cada vez más globalizado, cualquier manifestación de interés respecto del proceso político interno tiende a motivar respuestas concretas y repercute en la opinión pública del país afectado. Las mismas protestas de los regímenes afectados frente a la interferencia extranjera indican que este activismo tiene relevancia y desempeña una función valiosa. Todo indica que esta tendencia seguirá acentuándose en el futuro.

Ciertamente, no es indiferente la importancia y naturaleza de los actores externos que protagonizan estas acciones declarativas. No es lo mismo una expresión de interés más o menos constante procedente de los Estados Unidos que una declaración de un gobierno de un Estado pequeño. No es lo mismo una acción aislada que una concertación de esfuerzos de un número cada vez más amplio de gobiernos. También importa la autoridad política del país o de la institución que ejerce la acción. Por su parte, las ONG constituyen actores muy relevantes en este plano. Una denuncia de Amnesty International o de Americas Watch puede ser más efectiva que una declaración gubernamental o una resolución de una organización internacional. Lo mismo puede afirmarse sobre el papel de las grandes internacionales políticas y sindicales o bien de sus miembros más calificados. Muchos casos latinoamericanos revelan la gran influencia que puede ejercer la Iglesia Católica y, en menor medida, otras agrupaciones religiosas, en favor de los derechos humanos y de la democratización.

El factor personal también asume relevancia en este contexto. Las referencias que se hacen al papel desempeñado por ciertos embajadores de los Estados Unidos en casos como los de Chile o Portugal, pueden ser confirmadas por múltiples casos en América Central y el Caribe. Lo mismo puede afirmarse respecto de personalidades relevantes de la política, las artes o de otras actividades que gozan de estimación social en la región o en el mundo.

La acción declarativa tiene a menudo una importante vertiente multilateral, como la que ha ejercido las Naciones Unidas en determinadas situaciones, sea apoyando la Primavera 
Árabe en el Medio Oriente o la apertura iniciada en Myanmar, el Consejo de Europa y la Unión Europea, para el caso de los países que se sitúan en su periferia, o bien la OEA en casos como Haití u Honduras. La importancia de estas démarches diplomáticas no debe ser subestimada. Y si éstas tienen un carácter multilateral, el consenso de varios Estados les otorga una mayor representatividad internacional y abre el camino para una acción multilateral más directa y enérgica.

Una segunda categoría de acciones incluye las sanciones políticas y económicas. Las posibilidades en este terreno son múltiples. A nivel unilateral, ellas comprenden el retiro de representantes diplomáticos, la suspensión de ayuda económica o militar, la interrupción de suministros de armas o de repuestos, la ruptura de relaciones diplomáticas, la suspensión de un régimen de preferencias comerciales, el embargo comercial, sanciones a terceros que comercian con el régimen afectado, etc. A nivel multilateral, las sanciones pueden consistir en la interrupción de créditos por parte de organizaciones financieras internacionales, la suspensión de la participación del país afectado en una organización regional o mundial, la adopción de procedimientos de fiscalización en materia de derechos humanos, etc.

Las situaciones de ruptura constitucional o incumplimiento de normas mínimas de respeto democrático pueden también provocar sanciones. El debate sobre su efectividad ha sido siempre muy intenso. Aunque durante mucho tiempo hubo importantes dudas sobre la utilidad, la experiencia de casos tales como Rodesia-Zimbabwe, Sudáfrica, Namibia, Haití y otros tiende a contradecir las actitudes más escépticas frente a la eficacia de las sanciones. Se puede argüir que estos casos revisten un carácter extraordinario y que no se puede generalizar a partir de ellos. Pero, incluso aceptando esta reserva, no es aventurado suponer que las muy diversas sanciones aplicadas a otros casos, especialmente en América Latina, por lo menos han podido marcar una diferencia en el comportamiento de los regímenes autoritarios, han impuesto ciertos frenos a los arrestos autoritarios o han tenido un efecto disuasivo respecto de posibles amenazas involucionistas. Esta evaluación no implica asumir que las sanciones siempre alcanzan resultados positivos. Las medidas coercitivas deben ser analizadas cuidadosa y periódicamente en relación al contexto externo e interno en que se aplican y, por cierto, sólo pueden justificarse cuando se adoptan en un marco multilateral y con estricto apego a la legalidad internacional.

En una tercera categoría podríamos poner los incentivos de cooperación e integración. La posesión de un régimen democrático puede ser establecida como una condición indispensable para la participación de un país en un esquema de integración o de cooperación. En la experiencia europea, la posibilidad de adherir a un esquema de integración regional actuó como un poderoso incentivo de los procesos de democratización, en los casos de Grecia, España, Portugal y, posteriormente, los países de Europa Central y del Este. Cualquier interrupción del proceso democrático tendría un impacto enorme en la inserción económica externa del país afectado. En el caso de América Latina, los procesos de transición y de consolidación de la democracia que se generalizaron en la región durante los años 80 otorgaron un impulso a la integración y cooperación regional. Sin embargo, el menor desarrollo y el carácter incierto de varios de los procesos de integración hacen que la exigencia democrática tenga efectos menos dramáticos y, en los hechos, en el caso 
de Honduras, el golpe de Estado del 28 de junio de 2009 no tuvo el efecto de marginar a ese país del proceso de integración centroamericano.

Los países latinoamericanos han coincidido en la necesidad de introducir "cláusulas democráticas" en los nuevos instrumentos de cooperación que negocian entre ellos o con terceros países. Asimismo, la Unión Europea ha introducido estas cláusulas en los acuerdos de asociación que ha negociado o negocia con los países latinoamericanos. Se trata así de condicionar los beneficios del libre comercio y de la cooperación al mantenimiento de la democracia. Sin embargo, la evaluación de la situación de inconstitucionalidad está entregada a las mismas partes, sin que exista automatismo en la aplicación de la cláusula y sin que una entidad autónoma pueda evaluar si se cumplen o no las condiciones para su aplicación.

Una cuarta categoría de instrumentos incluye las políticas de apoyo, fortalecimiento e incentivos democráticos. Estas pueden ser aplicadas por actores estatales, Estados u organizaciones internacionales. La Alemania de postguerra, por ejemplo, desarrolló una política pionera en esta materia, fundamentalmente a través de sus fundaciones políticas, que están ligadas a cada una de las fuerzas políticas significativas en el país. De manera tardía, Estados Unidos trató de replicar ese modelo, creando sus propias fundaciones políticas, mientras que otros países, como Holanda, optaron por instituciones multipartidistas destinadas al mismo fin. Entre las organizaciones internacionales, se destaca el importante papel desarrollado por entidades regionales como el Consejo de Europa y la Organización de Estados Americanos (OEA) en el fortalecimiento de las instituciones democráticas en sus países miembros y en ocasiones en países terceros. Asimismo, en 1995 se estableció en Estocolmo el Instituto Internacional para la Democracia y la Asistencia Electoral, como una organización intergubernamental dedicada exclusivamente a la promoción de la democracia en el mundo. Por su parte, bajo el impulso de Estados Unidos, Polonia y otros países convocantes, el año 2000 se estableció la Comunidad de las Democracias, como una coalición de Estados democráticos destinada a fortalecer la democracia en el mundo. Especial mención merecen las tareas de observancia y supervisión de los procesos electorales, tarea que ha sido asumida con especial eficacia por organizaciones internacionales regionales como la OEA, o globales, como las propias Naciones Unidas ${ }^{16}$.

Una serie de entidades no estatales, tales como partidos políticos, internacionales políticas y sindicales, asociaciones sindicales, movimientos eclesiásticos, fundaciones y otras $\mathrm{ONG}$ de variado origen y naturaleza se han dedicado igualmente a apoyar el fortalecimiento de las instituciones democráticas en el mundo. Estos actores pueden ejecutar una serie muy amplia de acciones, que va desde la asistencia técnica a las fuerzas democráticas hasta la cooperación en la construcción de nuevas instituciones políticas, desde la formación de líderes hasta el apoyo económico de fuerzas y movimientos democráticos, desde la

16 Para Franck, la observancia internacional de elecciones constituía un elemento central de un nuevo régimen internacional en materia de democracia. Véase Thomas M. Franck, "Legitimacy and the democratic entitlement", en Fox y Roth, Democratic Governance, p. 35 y sgtes. Véase también Thomas Carothers, Aiding Democracy Abroad: The Learning Curve, Washington D.C.: Carnegie Endowment for International Peace, 1999. 
asesoría en campañas políticas hasta la supervisión de elecciones. Estos instrumentos han experimentado un desarrollo notable durante las últimas dos décadas.

\section{Dilemas y riesgos}

El régimen internacional para proteger y promover la democracia no está exento de dilemas y riesgos ${ }^{17}$. El primer dilema se plantea con respecto al principio de la no intervención en los asuntos internos de otros Estados, una de las piedras angulares del sistema internacional. La acción colectiva en favor de la restauración e incluso la defensa de la democracia pone en duda la noción de que la legitimidad política interna de un país corresponde a su jurisdicción exclusiva e introduce una importante excepción al principio clásico de la soberanía plena de los Estados. Por lo mismo, tanto el derecho internacional como diversos Estados mantienen importantes reservas frente a esta evolución. Está claro que la acción internacional en defensa de la democracia ya no está subordinada en términos absolutos al principio de la no intervención y que la relación entre ambos principios no tiene un carácter inmutable. Pero el derecho internacional recoge esta evolución de manera gradual ${ }^{18}$.

Es importante agregar que la acción internacional que comienza a ser legitimada en este ámbito asume un carácter multilateral y, en consecuencia, exige el apego a las normas y procedimientos fijados para estos efectos en las organizaciones internacionales, tanto globales como regionales, así como un consenso relativamente amplio por parte de la comunidad mundial. Son precisamente estas consideraciones las que permiten establecer un claro contraste entre el intervencionismo unilateral y la acción multilateral. Sólo esta última da pie para hablar de un régimen embrionario.

Un segundo dilema se refiere al concepto de democracia que está en la base de este nuevo activismo internacional o regional. No se trata tanto de revivir el viejo y distorsionado debate sobre el valor de la democracia formal, que marcó tan profundamente el pensamiento político latinoamericano durante los años 60 y 70 y que, afortunadamente en opinión de este autor, hoy parece superado. Tampoco se trata de tomar en serio las calificaciones interesadas que hacen los gobernantes sobre las diversas formas de democracia posibles y que han pretendido justificar la carencia de libertades básicas en regímenes muy dispares. Dejando a un lado consideraciones teóricas y filosóficas, la democracia puede ser definida con relativa claridad desde el punto de vista operacional. Ella incluye elecciones libres, secretas, informadas y competitivas, en que todos los ciudadanos adultos de un país pueden votar o ser votados. Implica la posibilidad real de alternancia en el poder, la existencia de un Estado de Derecho, de

17 Los párrafos siguientes se basan en Alberto van Klaveren, "La promoción de la democracia como principio de la política exterior de Chile”, publicado en Mario Artaza y César Ross (eds.). La política exterior de Chile, 1990 -2009. Del aislamiento a la integración global. Santiago: RIL editores 2012, pp. 115 -139

18 Véase el mesurado artículo de James Crawford, "Democracy and the body of international law", en Fox y Roth, Democratic Governance and International Law, pp. $91-120$. 
una división de poderes, de un poder judicial independiente y del libre juego entre el gobierno y una oposición que fiscaliza. Y, sobre todo, la democracia incluye la libertad de expresión, la libertad de asociación, el respeto al pluralismo, la libertad de prensa y otros derechos básicos. Descrita en esos términos, la democracia reúne los dos criterios que el destacado politólogo Robert Dahl considera como críticos: la posibilidad de oposición y la posibilidad de participación ${ }^{19}$.

El dilema se plantea en el sentido de que en varios regímenes surgidos de elecciones razonablemente libres persisten situaciones muy graves en materias de derechos humanos o incluso pueden derivar hacia modelos autoritarios. También puede producirse el caso de que la aplicación de los mecanismos para la defensa de la democracia no se desarrolle de una manera plenamente satisfactoria o no entregue los resultados esperados en términos de un proceso de verdadera consolidación democrática, como lamentablemente puede suceder en los Estados fallidos.

Asimismo, suele plantearse la necesidad de conciliar objetivos distintos y a veces contrapuestos de política exterior. Un objetivo tan loable como la defensa de la democracia no puede ser evaluado en forma aislada con respecto a otros intereses de la comunidad internacional como el mantenimiento de la paz y de la seguridad internacionales o la estabilidad en una cierta región. También puede surgir una contradicción entre intereses políticos y estratégicos. Esta última no afecta sólo a las grandes potencias, sino que también tiene vigencia en los ámbitos regionales. Guste o no guste, los atentados a la democracia en países vecinos suelen ser tratados con una natural cautela, con el fin de no interrumpir o afectar vínculos que se consideran fundamentales para una convivencia internacional razonable o para la defensa de intereses específicos de los Estados. La estabilidad de las relaciones vecinales puede llegar a primar sobre otras consideraciones, por legítimas que ellas sean.

Estas consideraciones nos remiten igualmente a la espinosa cuestión del "doble estándar”. ¿Corresponde aplicar el nuevo régimen para la defensa y promoción de la democracia en todos los casos? Cabe agregar que el problema del doble estándar no se deriva sólo de un problema de mayores o menores simpatías ideológicas. Está también la cuestión de la eficacia de la acción internacional. Resulta por lo menos dudoso que los partidarios de construir un régimen internacional mucho más fuerte para defender la democracia, que incluya una batería de sanciones que vayan desde el aislamiento diplomático y económico hasta la intervención internacional para restaurar la democracia, estén dispuestos a recomendar seriamente medidas drásticas para impulsar la democracia o hacer frente a una eventual interrupción de la democracia en los países mayores de una cierta región o bien a potencias económicas con las que se mantienen florecientes negocios. Aunque pueda resultar una conclusión cínica, siempre será más fácil aplicar sanciones enérgicas en este terreno a los países pequeńos y más débiles. Estas consideraciones explican el carácter embrionario y selectivo del régimen.

19 Robert A. Dahl, Polyarchy: Participation and Opposition. New Haven: Yale University Press, 1971. 


\section{Conclusión}

La promoción de la democracia ha sido y es una pieza clave de las políticas externas de una cantidad relevante de países en el mundo. Aunque se registran obvias diferencias en la intensidad, constancia e instrumentos que caracterizan a estas políticas, se puede observar una tendencia clara en esta materia, apoyada en una práctica que se ha extendido igualmente a organizaciones internacionales regionales $\mathrm{y}$, con ciertas reservas, globales.

Sin embargo, los dilemas planteados en la sección anterior obligan a una cierta cautela frente a las proclamaciones de un nuevo régimen internacional en este ámbito. La práctica frente a las rupturas democráticas no siempre ha sido consistente y las políticas de la comunidad internacional frente a los regímenes autoritarios han variado desde la sanción y el aislamiento hasta la cooperación más estrecha y las alianzas estratégicas y militares. Quizás ha sido esa práctica la que explica la prudencia con que los tribunales internacionales globales han tratado el tema de la democracia ${ }^{20}$.

El fin de la guerra fría, el éxito de los procesos de transición democrática o redemocratización en Europa y América Latina y el breve momento unipolar que le siguió alentaron un gran optimismo en esta materia durante los años 90 . Se generó la ilusión de que los Estados sólo podían ser legítimos en la medida en que actuaban en favor de sus ciudadanos y que respetaban sus derechos ${ }^{21}$. La soberanía pareció hacerse condicional al origen de sus gobiernos y a la observancia de los derechos de los gobernados. Sin embargo, el consenso en esta materia ha estado limitado a un número acotado de Estados, de raigambre liberal, ubicados principalmente en Europa, las Américas y Oceanía, con algunos seguidores en África y Asia. Se trata entonces de un consenso político parcial que por la misma razón que es parcial no puede transformarse en forma plena en un consenso legal internacional.

Cabe preguntarse si este consenso tiene un carácter creciente, como lo sostuvieron en los años 90 autores como Huntington ${ }^{22}$, o si la tendencia en esa dirección es menos clara de lo que parece.

Los regímenes internacionales no dependen de las preferencias políticas o valóricas. Más bien, responden a un cierto contexto internacional. En la medida en que la promoción de la democracia forma parte de una tradición política liberal y occidental, su universalización y transformación en un régimen global dependerá de la expansión de esa misma tradición al resto del mundo. Sin embargo, esta expansión no es evidente en la actualidad. Como lo afirma Kupchan, el modelo político occidental no está siendo universalizado y tampoco está siendo remplazado por un nuevo modelo o centro de

20 Véase el análisis de Crawford, op. cit, de la sentencia del caso Military and Paramilitary Activities in and against Nicaragua (Nicar. v. United States), de la Corte Internacional de Justicia, 1986, pp. 99-100.

21 Andrew Hurrell, On Global Order, Oxford: Oxford University Press, 2007, p. 154.

22 Samuel P. Huntington, The Third Wave: Democratization in the Late Twentieth Century. Norman, Ok: Univ. Oklahoma Press. 1991. 
gravedad. Más bien, el nuevo mundo será políticamente diverso y habrá distintas concepciones de lo que constituye un orden justo y legítimo ${ }^{23}$. En la medida en que se mantenga esta tendencia, resultará difícil hablar de un régimen internacional, preciso y efectivo, para la defensa de la democracia. Esta realidad marca una diferencia con los regímenes internacionales vigentes en materia de derechos humanos, cuyo rango es superior en el derecho internacional y que, al menos nominalmente, suscita un consenso universal mayor, al igual que fuertes consensos regionales.

Se podría argumentar que la dificultad de establecer un régimen global para la democracia puede ser compensada por la adopción de regímenes regionales en este ámbito, especialmente en Europa y en América. Sin duda, esa opción está abierta. Sin embargo, tampoco en esas regiones existe una uniformidad política total. Por cierto hay bases para sostenerla en el caso de la Unión Europea. Pero si ampliamos el círculo a la periferia europea, la uniformidad se hace menos clara. Una revisión somera de la membresía del Consejo de Europa o, más todavía, de la Organización para la Seguridad y Cooperación de Europa (OSCE) arroja dudas sobre las credenciales democráticas de algunos de los Estados Partes. Y en las Américas, la creciente diversidad política que se observa en América Latina plantea interrogantes respecto de la viabilidad de profundizar la Carta Democrática Interamericana o establecer alianzas basadas en los modelos políticos de los países.

La defensa y promoción de la democracia tiene plena validez como principio de política exterior. Y países como Chile tienen todo el derecho de mantenerlo. No obstante, un principio, por legítimo y atendible que sea, no llega a configurar por sí mismo un régimen internacional. En un contexto de multipolaridad y diversidad política, la defensa y promoción de la democracia debe conciliarse con el principio de no intervención, sin perjuicio de que éste ya no tiene el valor absoluto que se le otorgó en el pasado, así como con los principios del pluralismo y de la aceptación de la diversidad a nivel internacional. En suma, estamos frente a un régimen embrionario, cuya evolución dependerá de la evolución política que experimente la comunidad internacional.

23 Charles A. Kupchan, No One's World. The West, the rising rest, and the coming global turn. New York: Oxford University Press, 2012, p. x. 